\title{
Indicatori ambientali e modello DPSIR: valutazione dello stato ambientale
}

\author{
Francesca Luongo $^{1}$, Stefania Cusano ${ }^{1}$, and Alessia Chiapparrone ${ }^{1}$ \\ ${ }^{1}$ Affiliation not available
}

\begin{abstract}
L'analisi integrata degli aspetti socio-economici è eseguita mediante il modello DPSIR: uno strumento capace di valutare la catena causale che conduce all'alterazione ambientale, misurata attraverso gli indicatori ambientali. Questi sono organizzati in una logica di sistema in grado di rispondere a domande politiche e per supportare tutte le fasi decisionali, che hanno come obiettivo azioni sostenibili per l'ambiente. Per valutare lo stato ambientale, sociale ed economico del territorio in esame, con riferimento al settore turistico, nasce, nel 2000, il Progetto S19 "Calcolo della Capacità di Carico e della Sostenibilità del Parco Nazionale del Vesuvio", facente parte del Progetto Integrato Territoriale "Vesevo".
\end{abstract}

\section{Indicatori ambientali e Modello DPSIR}

Gli indicatori ambientali forniscono dati di sintesi in grado di guidare l'analisi sullo stato di un certo ambiente, di guidare processi decisionali in campo politico, economico e sociale verso forme di sfruttamento sostenibili del territorio ${ }^{1} \mathrm{e}$ di monitorare le risposte a tali decisioni ${ }^{2}$.

La rete delle Agenzie è composta dall'Istituto Superiore per la Protezione e la Ricerca Ambientale, ISPRA, e da Agenzia Regionale per la protezione dell'ambiente, ARPA, e provinciali, APPA. ${ }^{3}$ 
Il modello DPSIR ${ }^{45}$ è costituito da:

- determinanti

- pressioni

- stato

- impatti

- risposte

I vari elementi del modello costituiscono i nodi di un percorso circolare di politica ambientale che comprende la percezione dei problemi, la formulazione dei provvedimenti politici, il monitoraggio dell'ambiente e la valutazione dell'efficacia dei provvedimenti adottati. A tal fine si ricorre all'utilizzo di indicatori descrittivi e/o di indicatori di valutazione ${ }^{6}$ :

- Indicatori descrittivi: descrivono la situazione reale riguardo ai problemi ambientali. Sono espressi in unità fisiche (emissioni $\left.\mathrm{CO}_{2}\right)$

- Indicatori di prestazione o di efficacia: è il rapporto tra un risultato raggiunto e un obiettivo prestabilito in termini di politica ambientale (\% di rifiuti raccolti in modo differenziato/obiettivo di raccolta differenziata)

- Indicatori di efficienza: rapporto tra un risultato ambientale raggiunto e le risorse economiche impiegate per raggiungerlo (riduzione delle emissioni atmosferiche/ costo degli interventi strutturali e/o gestionali)

- Indicatori del benessere totale: insieme di indicatori che misurano la sostenibilità totale (ad esempio l'impronta ecologica) 


\section{Modello DPSIR: applicazione}

L'area protetta del Parco Nazionale del Vesuvio è stato oggetto di studio per poterne migliorare la "governance", con riferimento al settore turistico, e la qualità ambientale. ${ }^{7}$ Per questo motivo viene introdotto l'indice di sostenibilità ambientale, calcolato dopo aver individuato sei componenti ambientali e un set di indicatori ambientali per ognuna. Il valore di ciascun indicatore viene posizionato all'interno di una scala di qualità ambientale, da 0 (valore minimo di qualità) a 100 (valore massimo). Successivamente si individua un indice ambientale parziale relativo alla componente ambientale, la cui media aritmetica restituirà il valore dell' indice complessivo, necessario per la valutazione finale della qualità ambientale, tramite una scala che individua cinque classi di qualità ambientale.

La componente ambientale Aria è analizzata attraverso indicatori di stato che riguardano la concentrazione massima di una serie di inquinanti, confrontati con la normativa, e il numero di superamenti dei livelli di attenzione/allarme. In primo luogo si è ottenuto il rapporto tra concentrazione massima rilevata e limite normativo per ciascun inquinante; successivamente si è determinato il valore medio dei rapporti trovati; poi convertito in una scala di qualità ambientale.

In conclusione l'indice parziale relativo alla componente aria risulta pari a 36,8 , ovvero una situazione di scarsa qualità ambientale.

La componente 'Acqua' si basa sull'analisi dello stato qualitativo dei corpi idrici sotterranei e sulle caratteristiche chimiche dell'acqua ${ }^{8}{ }^{9}$. La valutazione è avvenuta secondo l'uso di classi qualitative (classi A, B, C, D) e chimiche (classi 1, 2, 3, 4, 0) esplicitate nel Decreto Legislativo 152/99. Nel caso in analisi siamo di fronte alle classi B e 3, arrivando a definire lo stato ambientale sufficiente con un valore di 67.

Gli indicatori utilizzati per la componente ambientale 'Suolo' e per 'Paesaggi ed Ecosistemi' sono valutati seguendo il medesimo iter lavorativo, il valore dell'indice parziale è pari rispettivamente 
a 49,93, collocando la componente Suolo in una posizione di qualità appena sufficiente, e a 67,74, qualificando i Paesaggi ed ecosistemi nella classe di buona qualità.

Riguardo all'analisi della componente Rifiuti si sono selezionati alcuni indicatori di pressione nonché la percentuale di rifiuti gestiti in raccolta differenziata e l'indice di gestione dei rifiuti urbani ${ }^{10}$. Le percentuali di rifiuti differenziati sono calcolate applicando la seguente formula per $\mathrm{i}$ vari comuni dell'area:

In definitiva il valore parziale, dato dalla media dei valori precedenti, è pari a 17,10 ed evidenzia che la situazione rifiuti è ad un livello insufficiente.

Con riferimento alla componente Traffico, si è costruito un indice specifico che valuti il numero di veicoli che sostano nell' area tramite il calcolo del numero di biglietti venduti al giorno per l'ascesa al cratere. Assegnando il valore di 20 e 10 per le auto e i pullman, il valore medio è pari a 15 , ovvero una classe insufficiente.

L'indice totale del Parco del Vesuvio è pari a 42,26, ottenuto dalla media tra gli indici parziali di ciascuna componente ambientale, e corrisponde ad una situazione di qualità ambientale complessiva appena sufficiente.

Riferendoci al modello DPSIR, dalla valutazione è emerso che l'area protetta presenta un forte grado di antropizzazione dovuto in particolare alla facile accessibilità, all'elevata densità edilizia, alla fruizione turistica di massa e al consumo del "bene parco" eccessivo. Questi ultimi generano un aumento di rifiuti e di emissioni, che alterano lo stato ambientale dei corpi idrici, dei paesaggi ed ecosistemi, del suolo e dell'aria, causando uno squilibrio del sistema ambientale. In risposta a queste problematiche, occorre correggere e tenere sotto controllo i valori degli indicatori, adottando una procedura di monitoraggio, che ha l'obiettivo di minimizzare i rifiuti prodotti dai vari 
comuni del parco e di diminuire i veicoli che accedono alla zona del cono, al fine di garantire il mantenimento di un equilibrio del sistema ambientale ${ }^{11}$.

\section{Conclusioni}

L'obiettivo del Progetto S19 è la valutazione della qualità ambientale del sistema del Parco Nazionale del Vesuvio. L'elaborazione di un sistema di indicatori e indici ambientali ha messo in luce la tolleranza delle diverse componenti ambientali alle pressioni antropiche, con particolare riferimento agli impatti prodotti dal settore turistico. In conclusione la qualità ambientale risulta essere appena sufficiente; ciò comporta risposte tempestive da parte delle amministrazioni attraverso piani di monitoraggio e scelte di pianificazione per assicurare l'equilibrio ambientale nel tempo ${ }^{12}$.

\section{References}

1.V.Naddeo, K. H. C., M.Balakrishnan. Frontiers in Water-Energy-Nexus-Nature-Based Solutions, Advanced Technologies and Best Practices for Environmental Sustainability - Proceedings of the 2nd WaterEnergyNEXUS Conference, November 2018, Salerno, Italy — Vincenzo Naddeo — Springer. Springer Nature 1, (2018).

2.Water-Energy Nexus: Evaluation of the Environmental Impact on the National and International Scenarios. Frontiers in Water-Energy-Nexus-Nature-Based Solutions, Advanced Technologies and Best Practices for Environmental Sustainability (2019).

3.Bo, F. Indicatori ambientali e loro rappresentazione grafica. in (Federico Bo, 2006).

4.Umbria, A. R. P. A. Il modello di riferimento DPSIR. in Relazione sullo stato dell'ambiente in Umbria (CRACE, 2004).

5.Mazzella, D. Linee guida per la redazione della relazione sullo stato per l'ambiente a livello 
territoriale. in Manuale e linee guida 72 (ISPRA, 2011).

6.Naddeo, V., Belgiorno, V., Zarra, T. \& Scannapieco, D. Dynamic and embedded evaluation procedure for strategic environmental assessment. Land Use Policy 31, 605-612 (2013).

7.Marta Bottero, E. C. Costruzione di un indice per la valutazione della sostenibilità ambientale di un'area protetta: il caso del Parco nazionale del Vesuvio.. in (XXIX Conferenza Italiana di scienze regionali., 2010).

8.V.Naddeo, K. H. C., M. Balakrishnan. Frontiers in Water-Energy-Nexus-Nature-Based Solutions, Advanced Technologies and Best Practices for Environmental Sustainability - Proceedings of the 2nd WaterEnergyNEXUS Conference, November 2018, Salerno, Italy - Vincenzo Naddeo - Springer. (Springer International Publishing, 2020).

9.Environmental technologies for the sustainable development of the water and energy sectors IWA Publishing. (IWA publishing, 2020).

10.Naddeo, V. \& Korshin, G. Water energy and waste: The great European deal for the environment. Science of The Total Environment 764, 142911 (2021).

11.Scannapieco, D., Naddeo, V. \& Belgiorno, V. Sustainable power plants: A support tool for the analysis of alternatives. Land Use Policy 36, 478-484 (2014).

12.A.Nesticò, V. N., C.Elia. Sustainability of urban regeneration projects: Novel selection model based on analytic network process and zero-one goal programming. Land Use Policy 99 99, (2020).

13.Errami, M. \& Garner, H. A tale of two citations. Nature 451, 397-399 (2008).

14.Bo, F. Indicatori ambientali e loro rappresentazione grafica. in (Federico Bo, 2006).

15.Baldizzone, G. Enciclopediambiente - Modello DPSIR. in Modello DPSIR (Giorgio Baldizzone). 


\section{Figure Captions}

Figure 1. Modello DPSIR (Fonte: Progetto S19)

Figure 2. Tabella 1: Indice di qualit@x@xtempboxaa@spacefactor $\Gamma 18 a \Gamma @ s p a c e$ factorambientaledellacomp ProgettoS19)

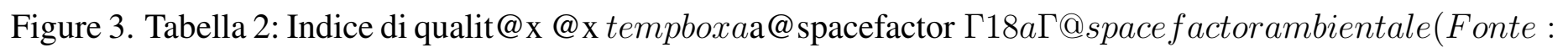
ProgettoS19) 


\section{Figures}

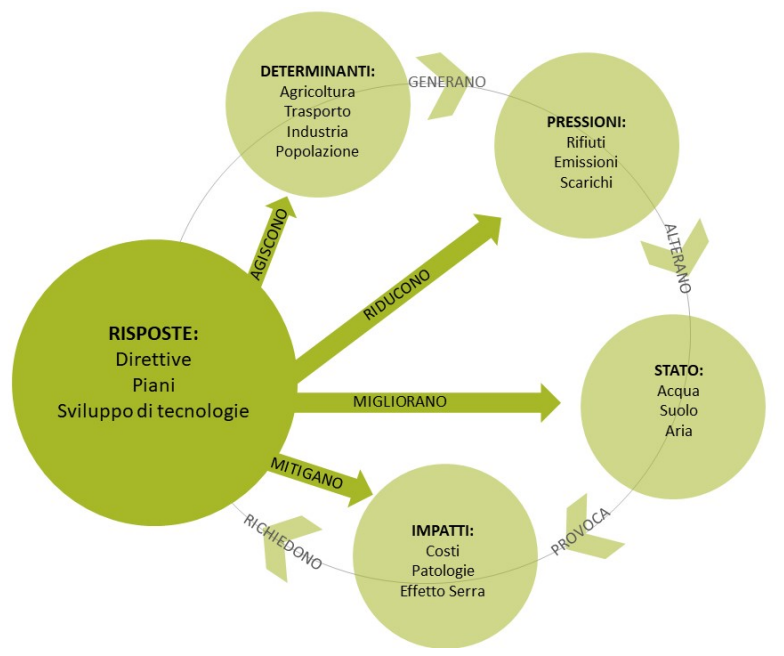

Figure 1: Modello DPSIR (Fonte: Progetto S19)

\begin{tabular}{|r|c|c|c|c|c|c|}
\hline \multicolumn{7}{|c|}{ Indice di qualità ambientale della componente aria } \\
\hline Indicatori & $\mathrm{CO}$ & $\mathrm{O}_{3}$ & $\mathrm{SO}_{2}$ & $\mathrm{NO}_{2}$ & $\mathrm{PTS}$ & $\mathrm{PM}_{10}$ \\
\hline Valore & 20,3 & 71,5 & 48,3 & 34,7 & 46 & 0 \\
\hline Valore medio & \multicolumn{7}{|c|}{36,8} \\
\hline
\end{tabular}

Figure 2: Tabella 1: Indice di qualit@x@x tempboxaa@spacefactor $\Gamma 18 a \Gamma @ s p a c e$ factorambientaledellacomp ProgettoS19)

$$
R D=\Sigma \frac{R D \text { di ogni comune }(\%) * \text { Numero di abitati del comune }}{\text { Tot abitati dell'area }}
$$




\begin{tabular}{|r|c|c|c|c|c|c|}
\hline \multicolumn{8}{|c|}{ Indice di qualità ambientale } \\
\hline Indici parziali & Aria & Acqua & Suolo & $\begin{array}{c}\text { Paesaggi ed } \\
\text { Ecosistemi }\end{array}$ & Rifiuti & Traffico \\
\hline Valore & 36,8 & 67 & 49,93 & 67,74 & 17,10 & 15 \\
\hline Valore medio & \multicolumn{7}{|c|}{42,26} \\
\hline
\end{tabular}

Figure 3: Tabella 2: Indice di qualit@ $@ @ \mathrm{x}$ tempboxaa @ spacefactor $\Gamma 18 a \Gamma @$ spacefactorambientale(Fonte : ProgettoS19) 